\title{
Moderation of Adult Depression by a Polymorphism in the FKBP5 Gene and Childhood Physical Abuse in the General Population
}

\author{
Katja Appel*,1,1 ', Christian Schwahn 2,8,10,1 ', Jessie Mahler', Andrea Schulz', Carsten Spitzer ${ }^{3}$, \\ Kristin Fenske, $^{4}$, Jan Stender ${ }^{4}$, Sven Barnow ${ }^{5}$, Ulrich John ${ }^{6}$, Alexander Teumer ${ }^{7}$, Reiner Biffar ${ }^{2}$, \\ Matthias Nauck ${ }^{8}$, Henry Völzke', Harald J Freyberger' and Hans J Grabe' \\ 'Department of Psychiatry and Psychotherapy, University of Greifswald, Greifswald, Germany; ${ }^{2}$ Department of Prosthetic Dentistry, \\ Gerostomatology and Dental Materials, Center of Oral Health, University of Greifswald, Greifswald, Germany; ${ }^{3}$ Department of Psychosomatic \\ Medicine and Psychotherapy, University of Hamburg, Hamburg, Germany; ${ }^{4}$ Department of Biological and Clinical Psychology, University of \\ Greifswald, Greifswald, Germany; ${ }^{5}$ Institute of Clinical Psychology, University of Heidelberg, Heidelberg, Germany; ${ }^{6}$ Institute of Epidemiology \\ and Social Medicine, University of Greifswald, Greifswald, Germany; ${ }^{7}$ Interfacultary Institute for Genetics and Functional Genomics, \\ University of Greifswald, Greifswald, Germany; ${ }^{8}$ Institute of Clinical Chemistry and Laboratory Medicine, University of Greifswald, Greifswald, \\ Germany; ${ }^{9}$ Institute for Community Medicine, University of Greifswald, Greifswald, Germany; ${ }^{10}$ Department of Mathematics and \\ Computer Science, University of Hamburg, Hamburg, Germany
}

\begin{abstract}
Childhood maltreatment and depressive disorders have both been associated with a dysregulation of the hypothalamic-pituitary-adrenal axis. The FKBP5 gene codes for a co-chaperone regulating the glucocorticoid-receptor sensitivity. Previous evidence suggests that subjects carrying the TT genotype of the FKBP5 gene single-nucleotide polymorphism (SNP) rsI360780 have an increased susceptibility to adverse effects of experimental stress. We therefore tested the hypothesis of an interaction of childhood abuse with rs 1360780 in predicting adult depression. In all, 2157 Caucasian subjects from the Study of Health in Pomerania (German general population) completed the Beck Depression Inventory (BDI-II) and Childhood Trauma Questionnaire. The DSM-IV diagnosis of major depressive disorder (MDD) was assessed by interview. Genotypes of rs 1360780 were taken from the Affymetrix Human SNP Array 6.0. Significant interaction ( $p=0.006$ ) of physical abuse with the TT genotype of rs I 360780 was found increasing the BDI-II score to I7.4 (95\% confidence interval $(\mathrm{Cl})=12.0-22.9)$ compared with 10.0 (8.2-1 I.7) in exposed CC/CT carriers. Likewise, the adjusted odds ratio for MDD in exposed TT carriers was $8.2(95 \% \mathrm{Cl}=1.9-35.0)$ compared with $1.3(0.8-2.3)$ in exposed subjects with CC/CT genotypes. Relative excess risk due to interaction (RERI) analyses confirmed a significant additive interaction effect $(\mathrm{RERI}=6.8 ; 95 \% \mathrm{Cl}=0.64-33.7$; $p<0.05)$. In explorative analyses, the most severe degree of sexual and emotional abuse also yielded significant interaction effects $(p<0.05)$. This study revealed interactions between physical abuse and rs 1360780 of the FKBP5 gene, confirming its role in the individual susceptibility to depression. Given the large effect sizes, rs 1360780 could be included into prediction models for depression in individuals exposed to childhood abuse.

Neuropsychopharmacology (201 I) 36, 1982-1991; doi: I0.1038/npp.201 I.8I; published online 8 June 20I I
\end{abstract}

Keywords: major depression; childhood abuse; general population; FKBP5 gene; gene-environment interaction; CTQ

\section{INTRODUCTION}

A dysfunction of the hypothalamic-pituitary-adrenal (HPA) axis has been suggested as an important pathogenetic factor in depressive disorders (Holsboer, 1999, 2000). Normalization of the HPA function was found to be a

*Correspondence: Dr K Appel, Department of Psychiatry, Ernst-MoritzArndt-University of Greifswald, Ellernholzstraße I-2, Greifswald 17475, Germany, Tel: + 49 (0)3834/86 6905, Fax: + 49 (0)3834/86 6889,

E-mail: katja.appel@uni-greifswald.de

IIThese authors contributed equally to this work.

Received 7 January 20 II; revised 5 April 201 I; accepted I2 April 201 I neuroendocrinological correlate of the clinical improvement of depression (Zobel et al, 2001). In search for the genetic and functional mechanisms underlying the HPA dysregulation in depression, recent research focused on genes involved in HPA axis regulation, such as the genes coding for the corticotrophin-releasing hormone receptor 1 (Bradley et al, 2008) and for FKBP5 (Binder et al, 2004, 2008; Binder, 2009).

The FKBP5 gene is located on chromosome 6p21 and consists of 10 exons. The FKBP5 gene codes for FK506binding protein 51 (FKBP5), a co-chaperone of hsp90, which regulates the glucocorticoid-receptor (GR) sensitivity (Binder, 2009). FKBP5 is mainly expressed in the brain, and 
in a broad array of human cells, including muscle, liver, and thymus tissue (Gawlik et al, 2006). Functionally, cortisol induces the FKBP5 expression by activation of glucocorticoid-response elements (Vermeer et al, 2003). In turn, FKBP5 binding to the GR reduces the GR affinity for cortisol and diminishes the amount of activated GR translocation to the cell nucleus (Wochnik et al, 2005).

Interestingly, common single-nucleotide polymorphisms (SNPs) within the FKBP5 gene have been found to increase the FKBP5 protein expression, for example, the highinduction allele $(\mathrm{T})$ of the FKBP5 gene SNP rs 1360780 (Binder et al, 2004). In line with the pathophysiological model, the high-induction alleles of the FKBP5 gene are associated with a relative GR resistance (Binder et al, 2008). Recently, it was demonstrated that healthy subjects homozygote for the high-induction alleles had a significantly slower recovery of stress-induced cortisol responses and higher levels of anxiety during the recovery phase of a psychosocial stress test than subjects with other genotypes (Ising et al, 2008). A corresponding finding was reported by Luijk et al (2010) when investigating the cortisol reactivity of 14-month-old infants during a stress test: the rs 1360780 $\mathrm{T}$ allele was directly associated with an increased cortisol response. Moreover, insecure-resistant attachment behavior interacted with rs1360780 $\mathrm{T}$ allele increasing the cortisol response.

Depressed patients homozygote for the high-induction allele $(\mathrm{T})$ of rs 1360780 showed a significantly faster response to antidepressant treatment and suffered from more previous episodes than the carriers of the CT or CC genotypes (Binder et al, 2004). In the STAR ${ }^{\star} \mathrm{D}$ study, only the CT genotype but not the $\mathrm{T}$ allele was associated with depression in White non-Hispanics compared with controls (Lekman et al, 2008). In some clinical samples with smaller size no association was found (Gawlik et al, 2006; Papiol et al, 2007).

Following the approach of gene-environment interactions (Caspi et al, 2003; Grabe et al, 2005, 2011), Binder et al (2008) investigated the interaction between SNPs of the FKBP5 gene and childhood abuse in adult posttraumatic stress disorder (PTSD). They found four highly linked SNPs of the FKBP5 gene (including rs1360780) that interacted significantly with childhood abuse to predict PTSD but not depression scores in adulthood. The interaction between SNPs of the FKBP5 gene (including rs1360780) and childhood adversity in PTSD was confirmed in African Americans but not in European Americans (Xie et al, 2010). A further study found interactions between SNPs of the FKBP5 gene (including rs1360780) and childhood trauma in adult suicide attempts; however, in this study, the common risk allele was protective (Roy et al, 2010). As some studies have suggested that early life stress precedes the HPA axis hyperactivity observed in major depressive disorder (MDD) (Heim and Nemeroff, 2001; Carpenter et al, 2004), an interaction between variations within the FKBP5 gene and childhood abuse could be of relevance in adult depression too.

We therefore investigated the hypothesis of an interaction between the high-induction TT genotype of rs 1360780 and childhood abuse and neglect in adult depression. This hypothesis was tested in a general adult population sample of 2157 subjects using the Beck Depression Inventory
(BDI-II) (Beck and Steer, 1987) score as the first outcome parameter. The BDI-II was chosen as primary outcome because of the higher statistical power of dimensional outcomes. Significant results were then replicated in a second model using DSM-IV (American Psychiatric Association, 1994) lifetime MDD as outcome variable. This was done because of two reasons: (1) to replicate and confirm the BDI-II interaction findings with a different but correlated outcome that assesses the diagnosis of lifetime occurrence of depression derived from an interview and (2) to evaluate the putative relevance of the interaction findings for clinical depression, which is of course different than the self-rated BDI-II score on depressive symptoms in the general population.

\section{MATERIALS AND METHODS}

\section{Sampling and Phenotyping Methods}

Sample and sample recruitment. Data from the Study of Health in Pomerania (SHIP) were used (John et al, 2001; Grabe et al, 2005). The target population was comprised of adult German residents in northeastern Germany living in three cities and 29 communities, with a total population of 212157 . A two-stage stratified cluster sample of adults aged 20-79 years (baseline) was randomly drawn from local population registries. The net sample (without migrated or deceased persons) comprised 6267 eligible subjects, of which 4308 Caucasian subjects participated at baseline SHIP-0 between 1997 and 2001. Follow-up examination (SHIP-1) was conducted 5 years after baseline and included 3300 subjects.

In 2007, the 'Life-Events and Gene-Environment Interaction in Depression' (LEGEND) study was started (Völzke et al, 2011). Until then, 639 participants from the baseline sample SHIP-0 were either deceased $(n=383)$ or refused further participation $(n=256)$. Thus, 3669 participants were invited to take part in the LEGEND study. During the conduction of LEGEND (June 2007 until August 2010), 92 participants were deceased and 1011 subjects refused participation in the LEGEND study. In all, 132 subjects did not respond to repeated efforts of contact (at least three written invitations, 10 telephone calls, and five home visits). Thirty-five subjects agreed to participate but missed all appointments.

Among the 2400 subjects who participated in the LEGEND study, 134 subjects were excluded from the analyses because of unreliable information or inconsistencies in the interview according to the judgment of the interviewer and the supervisor. Additionally, 47 subjects were excluded because of missing genetic data, 60 because of missing BDI-II values $(n=45)$ or MDD diagnosis $(n=19)$, and two because of missing data on the number of adult traumata. Thus, 2157 were eligible for the present gene-environment interaction analyses (Table 1).

There were expected differences between the subjects of the LEGEND study $(n=2400)$ and the other subjects from baseline (SHIP-0) regarding age $(p<0.001)$ and sex $(p=0.02)$, with the LEGEND subjects being younger and preferentially female (52.4\%). Importantly, based on the DSM-IV, stem questions for depression (Wittchen et al, 1999), which were implemented in SHIP-0 (John et al, 
Table I Descriptive Statistics of the Sample (Mean \pm SD or Number and Percentages Are Given)

\begin{tabular}{|c|c|c|c|c|c|c|c|c|c|}
\hline \multirow{2}{*}{ Characteristic } & \multicolumn{2}{|c|}{ Participants } & \multicolumn{2}{|c|}{ BDI-II } & \multicolumn{5}{|c|}{ MDD $^{c}$} \\
\hline & $\mathbf{N}$ & $\%$ & & $P$-value & Yes & $\%$ & No & $\%$ & $P$-value \\
\hline Age, years & 2157 & & $55.8 \pm 13.8$ & & $53.2 \pm 12.7$ & & $56.4 \pm 14.0$ & & $<0.001$ \\
\hline Age, range & 2157 & & $29.4-89.8$ & & $29.4-89.8$ & & $30.0-85.3$ & & \\
\hline Male & 1021 & 47.3 & $5.7 \pm 6.3$ & $<0.001$ & 117 & 31.6 & 904 & 50.6 & $<0.001$ \\
\hline Female & 1136 & 52.7 & $7.0 \pm 7.9$ & & 253 & 68.4 & 883 & 49.4 & \\
\hline \multicolumn{10}{|l|}{ (School) education ${ }^{d}$} \\
\hline$<10$ years/elementary school & 629 & 30.3 & $7.4 \pm 7.0$ & & 79 & 22.4 & 550 & 31.9 & \\
\hline 10 years/junior high & 1067 & 51.4 & $6.0 \pm 7.4$ & $<0.001$ & 200 & 56.8 & 867 & 50.3 & 0.003 \\
\hline Education & 30 & 1.4 & $6.6 \pm 8.3$ & & 9 & 2.6 & 21 & 1.2 & \\
\hline Unemployed & 246 & 11.8 & $7.5 \pm 8.5$ & & 40 & 11.4 & 206 & 11.9 & \\
\hline Housewife/maternity leave & 40 & 1.9 & $8.0 \pm 7.6$ & $<0.001$ & 6 & 1.7 & 34 & 2.0 & 0.215 \\
\hline Retired & 634 & 30.5 & $7.6 \pm 6.8$ & & 94 & 26.7 & 540 & 31.3 & \\
\hline Part-time employed & 227 & 10.9 & $5.7 \pm 6.6$ & & 44 & 12.5 & 183 & 10.6 & \\
\hline Fully employed & 899 & 43.3 & $5.2 \pm 6.7$ & & 159 & 45.2 & 740 & 42.9 & \\
\hline \multicolumn{10}{|l|}{ Social welfare ${ }^{d}$} \\
\hline No & 2063 & 99.4 & $6.3 \pm 7.1$ & 0.034 & 348 & 98.9 & 1715 & 99.5 & 0.254 \\
\hline Yes & 13 & 0.6 & $13.3 \pm 10.6$ & & 4 & 1.1 & 9 & 0.5 & \\
\hline Single, never married & 265 & 12.3 & $5.9 \pm 6.8$ & & 48 & 13.0 & 217 & 12.2 & \\
\hline Married & 1462 & 68.0 & $5.9 \pm 6.8$ & $<0.001$ & 204 & 55.4 & 1258 & 70.6 & $<0.001$ \\
\hline Divorced/married but living separately & 242 & 11.3 & $8.0 \pm 9.1$ & & 71 & 19.3 & 171 & 9.6 & \\
\hline Widowed & 182 & 8.5 & $8.7 \pm 7.7$ & & 45 & 12.2 & 137 & 7.7 & \\
\hline Number of traumata & 2157 & & & & 370 & & 1787 & & \\
\hline Mean \pm SD & & $0.76 \pm 0.96$ & & & $0.99 \pm 1.07$ & & $0.72 \pm 0.92$ & & \\
\hline Ist quartile-median - 3rd quartile & & $0-1-1$ & & & $0-1-1$ & & $0-0-1$ & & \\
\hline Minimum-maximum & & $0-7$ & & & $0-7$ & & $0-5$ & & \\
\hline No trauma & 1048 & 48.6 & $5.4 \pm 6.5$ & $<0.001$ & 137 & 37.0 & 911 & 51.0 & $<0.00$ । \\
\hline At least one trauma & 1109 & 51.4 & $7.3 \pm 7.7$ & & 233 & 63.0 & 876 & 49.0 & \\
\hline \multicolumn{10}{|l|}{ Posttraumatic stress disorder ${ }^{d}$} \\
\hline No & 2128 & 98.7 & $6.2 \pm 7.0$ & $<0.001$ & 353 & 95.4 & 1775 & 99.4 & $<0.00$ । \\
\hline Yes & 27 & 1.3 & $|8.1 \pm| 4.1$ & & 17 & 4.6 & 10 & 0.6 & \\
\hline \multicolumn{10}{|l|}{ Physical abuse ${ }^{d}$} \\
\hline No & 2047 & 95.5 & $6.2 \pm 7.0$ & $<0.001$ & 340 & 92.6 & 1707 & 96.1 & 0.008 \\
\hline Yes & 97 & 4.5 & $11.0 \pm 10.3$ & & 27 & 7.4 & 70 & 3.9 & \\
\hline \multicolumn{10}{|l|}{ Emotional abuse ${ }^{d}$} \\
\hline No & 2062 & 96.4 & $6.1 \pm 6.9$ & $<0.00$ I & 332 & 90.2 & 1730 & 97.7 & $<0.00$ । \\
\hline Yes & 76 & 3.6 & $14.5 \pm 11.3$ & & 36 & 9.8 & 40 & 2.3 & \\
\hline
\end{tabular}


Table I Continued

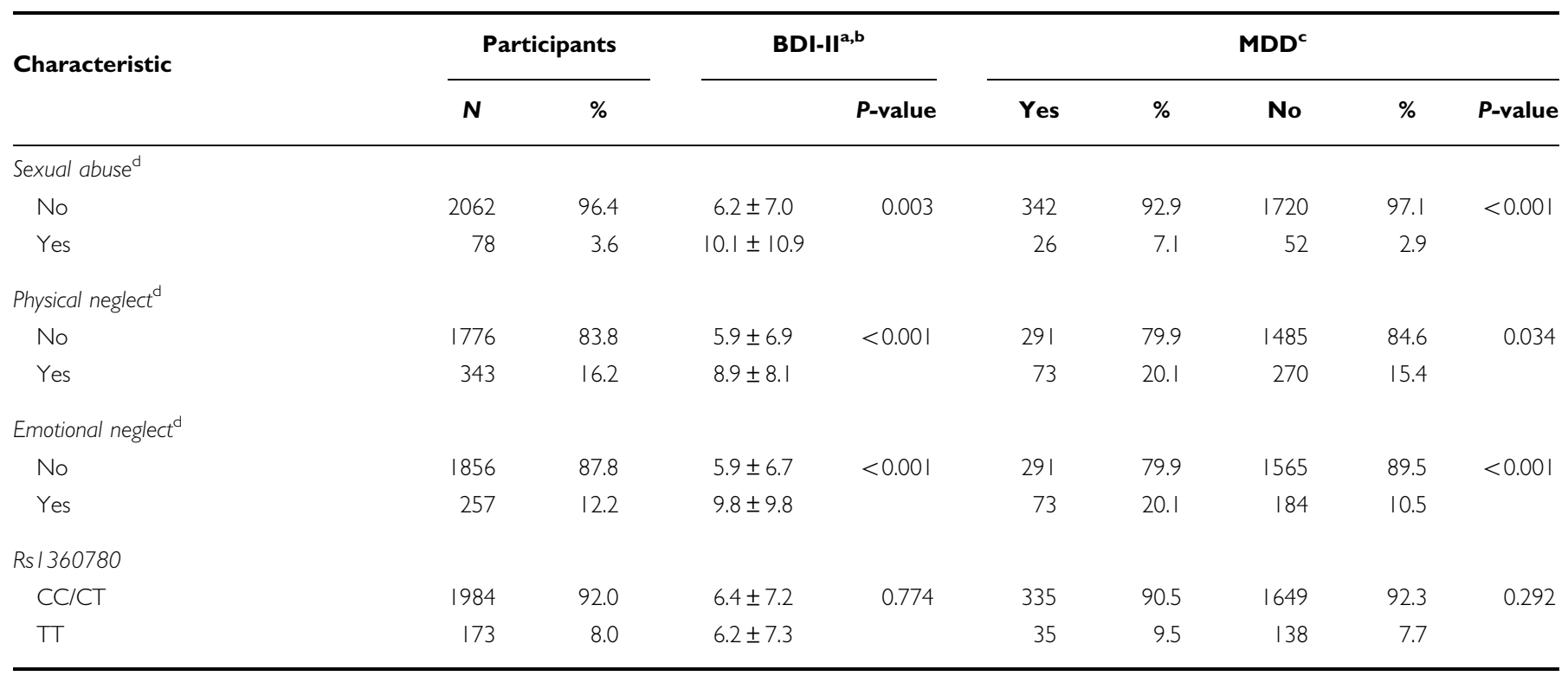

a For BDI-II comparing two groups, the observed means and SD were compared using t-test for equal or unequal variances, respectively.

${ }^{b}$ For BDI-II comparing more than two groups, the observed means and SD were compared using analysis of variance for equal variances and Kruskall-Wallis test for unequal variances.

${ }^{\mathrm{c}}$ For MDD, the observed numbers were tested for homogeneity using Fisher's exact test.

${ }^{\mathrm{d}} N<2157$ because of missing values.

2001), no differences emerged between the subjects from LEGEND $(n=2400)$ and the other subjects at (SHIP-0) $(p>0.5)$.

Because of some missing values in the Childhood Trauma Questionnaire (CTQ) (Bernstein et al, 2003), physical abuse could be analyzed in 2144 subjects, emotional abuse in 2138 subjects, sexual abuse in 2140 subjects, physical neglect in 2119 subjects, and emotional neglect in 2113 subjects. All participants gave written informed consent. SHIP and LEGEND were approved by the local institutional review board and conformed to the principles of the Declaration of Helsinki.

Phenotype measures. Current depressive symptoms were assessed with the BDI-II, a 21-item self-report questionnaire with high reliability and validity (Beck and Steer, 1987). The BDI-II was used as continuous outcome measure in all analyses. The distribution of the BDI-II scores dependent on the different qualities and levels of childhood trauma exposure is given in Table 2. The CTQ was used for selfreport of childhood maltreatment including emotional, sexual, and physical abuse as well as emotional and physical neglect (Bernstein et al, 2003). It has a total of 28 items rated on a 5-point Likert scale with higher scores indicating higher exposure to traumatic experiences. In addition to a dimensional scoring procedure, the manual provides threshold scores to determine the severity of abuse and neglect (none $=0$, low $=1$, moderate $=2$, and severe-toextreme $=3$ ). In independent studies, the CTQ was reported to have good reliability and validity; additionally, the 5 -factor model (ie the five subscales reflecting the different types of childhood trauma) has been empirically confirmed (Bernstein et al, 2003; Wingenfeld et al, 2010). Dichotomized variables ( 0 and 1 vs 2 and 3 ) were created for each dimension. To exploratively analyze the putative effects of higher childhood exposure on GxE, a second set of analyses was calculated testing the five CTQ dimensions for none (reference category) vs mild or moderate or severe, respectively. To further explore the impact of increasing severity/frequency of abusive events, a cumulative variable comprising only subjects with at least one severe traumatic category of emotional, physical, and sexual abuse was created (overall abuse severe category).

To evaluate lifetime diagnosis of MDD and PTSD, the Munich-Composite International Diagnostic Interview (M-CIDI) was applied (Wittchen and Pfister, 1997). The M-CIDI is a standardized fully structured instrument for assessing psychiatric disorders over the lifespan according to DSM-IV criteria. The computerized version of the interview was used by clinically experienced interviewers (psychologists) in a face-to-face situation.

All interviewers had undergone intensive and continuous training in the diagnostic procedures. In all, $84 \%$ of the subjects agreed to audiorecording of their interviews. In weekly meetings, the interviewers were supervised, and in difficult cases, consensus diagnoses were made by clinical experts (HJG, CS, HJF). For quality and training reasons, one randomly selected interview audiotape was double checked by two interviewers every 2 weeks.

Multiple studies regarding clinical validity and reliability of the M-CIDI had yielded excellent results for the inter-rater reliability and confirmed high clinical validity. Test-retest reliability analyses of the diagnosis of MDDs revealed $\kappa$-values between 0.62 and 0.77 , for the diagnosis of PTSD $\kappa$ was 0.72 (Wittchen and Pfister, 1997). Likewise, the interviewer $(n=17)$ had no impact on the lifetime diagnosis of MDD (logistic regression: odds ratio $(\mathrm{OR})=1, p=0.87, N=2247$ ) or PTSD in our study (OR $=1, p=0.97, N=2247$ ).

In order to adjust for the putative impact of adult traumata on the interaction effects, a variable comprising 
Table 2 Estimated Means $(95 \% \mathrm{Cl}$ ) of BDI-II Values Across Groups of the Putative Interaction Between the rs 360780 Genotype and Environment Factor (None, Mild vs Moderate, Severe) Adjusting for Sex, Age, and Number of Adult Traumata

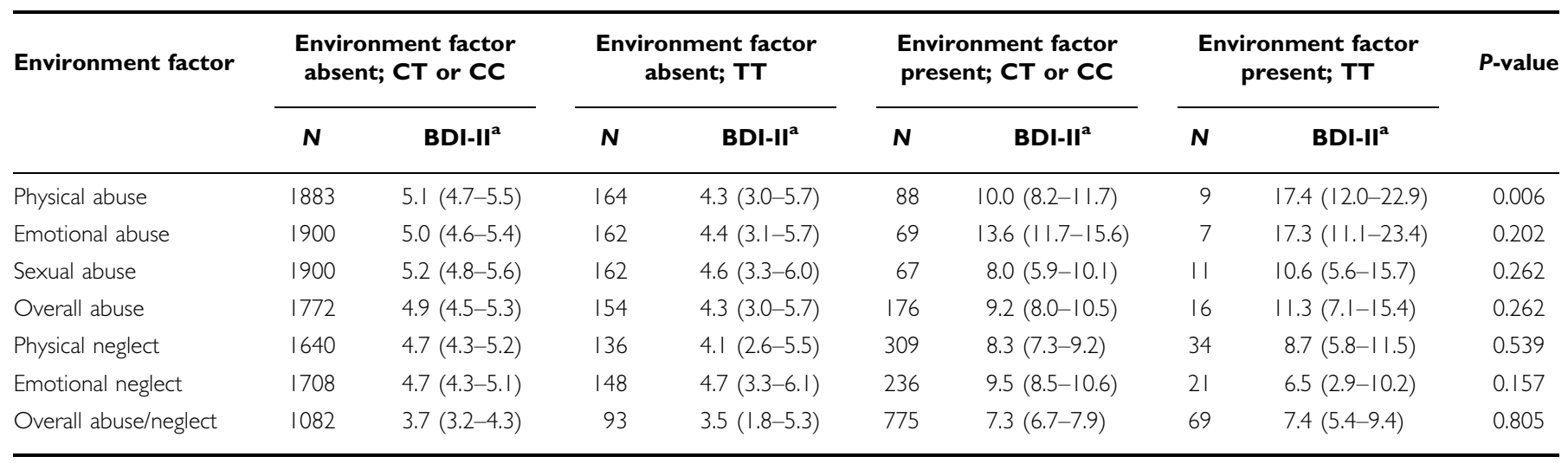

aEstimated means $(95 \% \mathrm{Cl})$ adjusted for age, sex, and number of adult traumata calculated with Tobit regression analysis.

the number of adult traumata was generated based on the trauma list of the PTSD section of the diagnostic interview (DSM-IV). All traumatic items $(n=10)$ except childhood sexual abuse were included into this adult trauma variable.

\section{Genetic Methods}

Genotyping. The SHIP sample was genotyped using the Affymetrix Human SNP Array 6.0. Hybridization of genomic DNA was done in accordance with the manufacturer's standard recommendations. The genetic data analysis workflow was created using the software InforSense. Genetic data were stored using the database Caché (InterSystems). Genotypes were determined using the Birdseed2 clustering algorithm. For quality control purposes, several control samples where added. On the chip level, only subjects with a genotyping rate on QC probesets (QC call rate) of at least $86 \%$ were included. Finally, all arrays had a sample call rate $>92 \%$. The overall genotyping efficiency of the GWA was $98.55 \%$. The genotype rate for the FKBP5 gene SNP rs1360780 was 99.6\%. Hardy-Weinberg equilibrium (HWE) of rs 1360780 was $p=0.080$ in SHIP-0 and $p=0.029$ in SHIP-LEGEND. The difference in allele frequencies between the SHIP-0 $(\mathrm{CC}=48.9 \%, \mathrm{CT}=42.9 \%, \mathrm{TT}=8.2 \%)$ and the LEGEND sample $(\mathrm{CC}=48.0 \%, \mathrm{CT}=44.0 \%, \mathrm{TT}=8.0 \%)$ was very small, indicating that a selection bias with respect to the genotype was not introduced by LEGEND.

For secondary analyses, 11 additional SNPs of the FKBP5 gene were identified that were genotyped by the Affymetrix Human SNP Array 6.0. However, only three SNPs met the following criteria: call rate $\geqslant 95 \%, \mathrm{HWE}>0.01$ and MAF $\geqslant 0.1$ (Supplementary Table S1a and b). A LD plot is given as Supplementary Figure S1 (chromosomal position: Supplementary Figure S2).

SNPs were used as basis for generating the multidimensional scaling (MDS) plot that were available in both the HapMap III r3 data set and in the imputed data set of the SHIP cohort, fulfilling the following QC criteria: HWE test $p$-value $>10^{-6}$, SNP call rate $>95 \%$, minor allele frequency $>1 \%$. Finally, 494375 SNPs went into the analysis. The MDS components were calculated using
PLINK and the results were plotted in R (Supplementary Figure S3).

\section{Statistical Analyses}

First, interaction analyses were performed between rs1360780 (TT vs TC and CC) and the five dimensions of the CTQ separately for BDI-II as dependent variable. The two-sided $\alpha$-level of 0.05 was therefore corrected to $0.05 /$ $5=0.01$ for the five environment factors analyzed.

Explorative analyses of the continuous BDI-II scores showed that the distribution was censored: $20.5 \%$ of the sample had BDI-II scores of zero. Therefore, we used Tobit regression, a technique designed to deal with censored distributions (Persons and Perloff, 1989). The direct effect of rs1360780 on BDI-II scores were also tested with Tobit regression.

Second, in order to enhance the validity of our positive findings, we chose an additional outcome: statistically significant interactions between CTQ dimensions and rs1360780 (TT vs TC and CC) in the BDI Tobit regression model were tested in a second model using the interviewbased diagnosis of lifetime MDD according to DSM-IV (American Psychiatric Association, 1994) as categorical outcome. Conventional logistic regression analyses were calculated and the results were expressed as ORs. Genotype differences in susceptibility to childhood abuse were estimated by biologic interaction, which is measured by departure from an additive model (Rothman, 2002). For the quantification of the magnitude of an interaction effect, the Relative Excess Risk due to Interaction (RERI) was calculated (Greenland et al, 2008; Grabe et al, 2009). The adjusted RERI is easily estimated using the basic formula for RERI (Rothman, 2002), as follows (OR= odds ratio):

$$
\begin{aligned}
\mathrm{RERI}= & \mathrm{OR}_{\mathrm{TT} \text { genotype and abuse }}-\mathrm{OR}_{\mathrm{TT} \text { genotype and no abuse }} \\
& -\mathrm{OR}_{\mathrm{CC} / \mathrm{CT} \text { genotypes and abuse }}+1
\end{aligned}
$$

For these confirmatory RERI analyses using MDD, we also applied a 95\% confidence interval (CI). If the lower bound of $95 \%$ CI of RERI was $\geqslant 0$, then the two-sided $p$-value for the interaction is significant on an $\alpha$-level of 0.05 . 
We first used a newly developed modified bootstrap approach to calculate an appropriate CI for RERI. This method was recommended by Nie et al (2010) to handle low cell counts. This modified approach is based on regularized instead of conventional logistic regression (see Supplementary Materials) and is supposed to yield narrower CIs compared with Zou's (Zou, 2008) formula (Nie et al, 2010). We additionally calculated the more commonly used formula by Zou. This formula yielded the identical results compared with modified bootstrap approach according to Nie. As Zou's method is more widely used, we report RERI results based on this method.

All analyses were performed as unadjusted and as adjusted for gender and age and the number of adult traumata. Because age was not linearly related to the outcomes, we ensured an adequate model fit (Hosmer and Lemeshow, 2000) choosing restricted cubic splines (Harrell, 2001), which are superior to categorized age groups (Greenland, 2008). To assess the effect of single observations (exposed subjects with MDD) on the statistic of interest, we performed detailed regression diagnostics (Hosmer and Lemeshow, 2000).

All analyses were performed with STATA/MP software, version 10.1 (StataCorp LP, College Station, TX, USA) and, if necessary, we used the free-ware $\mathrm{R}$, version 2.11.1 (Team, 2010) using the packages 'glmnet', 'Design' (Harrell, 2001) and 'boot' (see Supplementary Methods).

\section{RESULTS}

The sample characteristics are shown in Table 1. No direct effects of the TT genotype of rs1360780 on the BDI-II or MDD were found ( $p=0.25$, adjusted for sex and age). No direct association was found between the five subdimensions of the CTQ and rs1360780 (Fisher's exact test: $p>0.1$ ).

\section{Interaction Analysis for BDI-II}

The results of the interaction analyses between the five CTQ dimensions (none, mild $v s$ moderate, severe) and the TT $v s$ the TC/CC genotypes with regard to BDI-II scores are shown in Table 2. Tobit regression analyses revealed a significant interaction between the TT genotype and physical abuse regarding depressive symptoms $(p=0.006)$. However, also for emotional abuse and sexual abuse, relevant effect sizes were obtained that failed to reach statistical significance. The interaction effects for both neglect dimensions were small to absent, respectively. In Figure 1, the adjusted BDI-II mean scores dependent on the three genotypes of rs1360780 and the exposure to physical abuse are indicated. When subjects with PTSD $(n=29)$ were excluded from analyses, the interaction effect was almost unchanged $(p=0.011)$. When adjusting for PTSD, the interaction effect was reduced, but remained significant $(p=0.024)$ (see Supplementary Table S2).

The results of the explorative interaction analyses between the five CTQ dimensions testing none (reference category) vs mild or moderate or severe (separately) and the CC/CT vs the TT genotypes with regard to BDI-II scores are shown in Supplementary Table S3a and b. Tobit regression analyses revealed a significant interaction between the TT genotype and emotional $(p=0.048)$ and sexual abuse

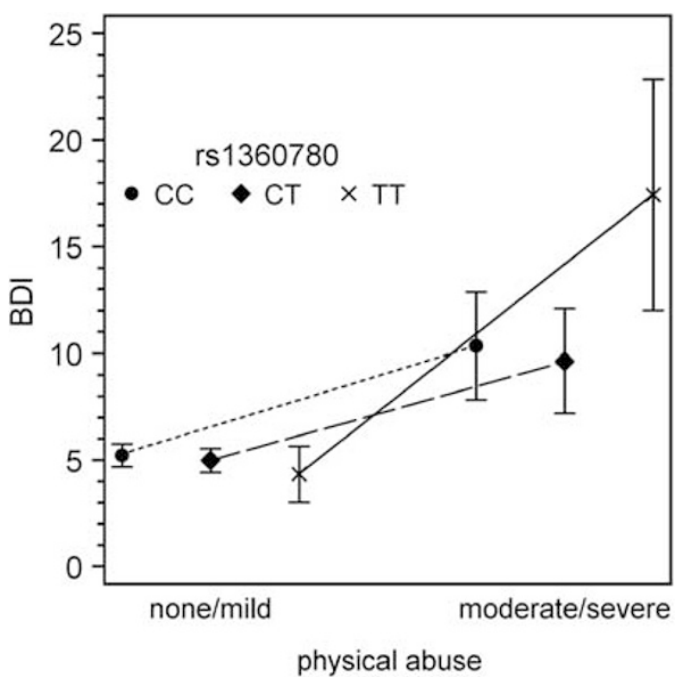

Figure I BDI-II mean scores (95\% Cls; adjusted for age and gender) dependent on the three genotypes of rs/360780 and the exposure to physical abuse (Tobit regression analysis).

$(p=0.004)$ but not for the neglect dimensions $(p>0.3)$ in the none $v s$ severe condition.

To further explore the impact of increasing severity/ frequency of abusive events, a cumulative variable comprising only subjects with at least one severe ( $v s$ none, mild, moderate) traumatic category of emotional, physical, and sexual abuse was created (Supplementary Table S4). A significant interaction between this overall abuse variable and the CC/CT $v s$ TT genotype emerged $(p=0.007)$.

In Supplementary Table S1a, the secondary interaction results for the additional SNPs rs7757037, rs4713899, and rs9368881 are given for each genotype separately. The SNPs (rs4713899 and rs9368881) linked to rs1360780 showed comparable results. However, no other SNP was superior to rs1360780 (rs4713899 had $n=1$ in the TT genotype/exposed group). Rs7757037 $\left(r^{2}=0.09\right.$ to $\left.\mathrm{rs} 1360780\right)$ showed no evidence of interaction.

\section{Interaction Analysis for MDD}

The positive interaction between the TT genotype and physical abuse was further investigated with regard to the diagnosis of MDD. In logistic regression analyses (Table 3), statistically significant effects were obtained for the combination of the TT genotype with physical abuse and the prevalence of lifetime MDD (adj. OR $=8.2 ; 95 \%$ $\mathrm{CI}=1.9-35.0$ ). In Figure 2, the adjusted OR for MDD dependent on the three genotypes of rs1360780 and the exposure to physical abuse are presented.

The interaction analysis for MDD indicated statistically significant effects $(p<0.05)$ for the unadjusted RERI of $8.4(95 \% \mathrm{CI}=0.78-39.0)$ and fully adjusted RERI of 6.8 (95\% CI $=0.46-33.7)$.

When excluding cases with PTSD, the combination of the TT genotype with physical abuse showed an adjusted $\mathrm{OR}=13.0(95 \% \mathrm{CI}=2.4-70.6)$ for lifetime MDD. The corresponding adjusted RERI was $12.0 \quad(95 \% \quad \mathrm{CI}=1.3-69.5)$. Adjusting the analyses also for PTSD (no exclusion), a RERI of $5.8(95 \% \mathrm{CI}=0.3-30.8)$ was obtained. 
Table 3 Interaction Between Physical Abuse and rs| 360780 for MDD

\begin{tabular}{|c|c|c|c|c|c|c|c|c|c|c|}
\hline \multirow{3}{*}{$\begin{array}{l}\text { Exposed to } \\
\text { physical abuse }\end{array}$} & \multirow[t]{3}{*}{ Genotype } & \multirow{3}{*}{$\begin{array}{c}\text { Subjects } \\
\text { with MDD } \\
\text { N }\end{array}$} & \multirow{3}{*}{$\begin{array}{c}\text { Subjects } \\
\text { without MDD } \\
\mathbf{N}\end{array}$} & \multirow{3}{*}{$\begin{array}{l}\text { MDD } \\
\text { risk (\%) }\end{array}$} & \multicolumn{6}{|c|}{ Analysis (logistic regression) } \\
\hline & & & & & \multicolumn{2}{|c|}{$\begin{array}{c}\text { Not } \\
\text { adjusted }\end{array}$} & \multicolumn{2}{|c|}{$\begin{array}{l}\text { Adjusted for age } \\
\text { and sex }\end{array}$} & \multicolumn{2}{|c|}{$\begin{array}{l}\text { Adjusted for age, sex, and } \\
\text { number of adult traumata }\end{array}$} \\
\hline & & & & & $\begin{array}{l}\text { Odds } \\
\text { ratio }\end{array}$ & $\begin{array}{c}95 \% \\
\mathrm{Cl}\end{array}$ & $\begin{array}{l}\text { Odds } \\
\text { ratio }\end{array}$ & $\begin{array}{c}95 \% \\
\text { Cl }\end{array}$ & $\begin{array}{l}\text { Odds } \\
\text { ratio }\end{array}$ & $\begin{array}{c}95 \% \\
\mathrm{Cl}\end{array}$ \\
\hline No & $\pi$ & 29 & 135 & 17.7 & 1.1 & $0.7-1.7$ & I.I & $0.7-1.7$ & 1.0 & $0.7-1.6$ \\
\hline Yes & $\mathrm{CC}$ or $\mathrm{CT}$ & 21 & 67 & 23.9 & 1.6 & $1.0-2.6$ & 1.5 & $0.9-2.6$ & 1.3 & $0.8-2.3$ \\
\hline Yes & $\Pi$ & 6 & 3 & 66.7 & $10.1^{\mathrm{a}}$ & $2.5-40.6$ & $8.7^{b}$ & $2.1-36.0$ & $8.2^{c}$ & 1.9-35.0 \\
\hline RERI 95\% & & & & & 8.4 & $0.78-39.0$ & 7.05 & $0.37-34.3$ & 6.80 & $0.46-33.7$ \\
\hline
\end{tabular}

${ }^{2}$ Data analysis for exact estimator is as follows: odds ratio $=10.1(95 \% \mathrm{Cl}=2.1-62.7)$; data analysis for jackknife estimator is as follows: odds ratio $=10.1$ (95\% $\mathrm{Cl}=2.0-5 \mid .9)$.

${ }^{b}$ Data analysis for jackknife estimator is as follows: odds ratio $=8.7(95 \% \mathrm{Cl}=1.8-43.1)$.

'Data analysis for jackknife estimator is as follows: odds ratio $=8.2(95 \% \mathrm{Cl}=1.8-38.0)$.

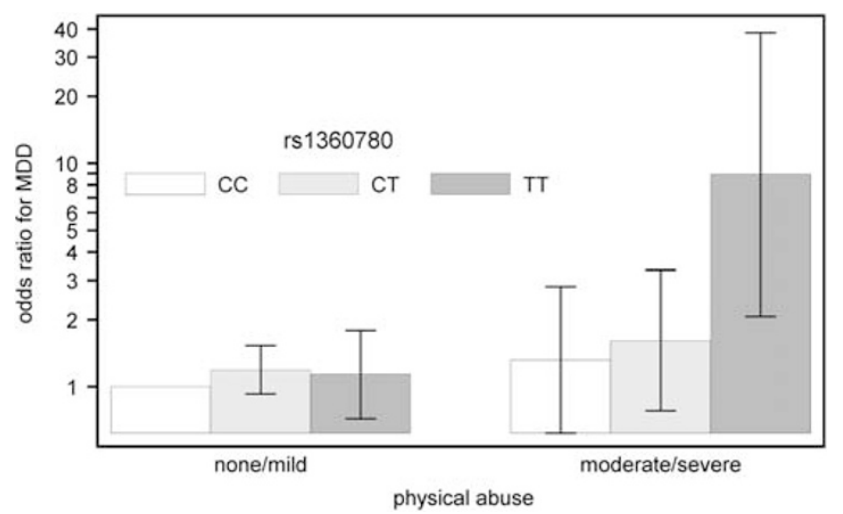

Figure 2 Odds ratios for MDD (95\% Cls, adjusted for age and gender) dependent on the three genotypes of rs 1360780 and the exposure to physical abuse (logistic regression analysis).

\section{DISCUSSION}

We identified an interaction between the TT genotype of rs1360780 and childhood physical abuse on the severity of present adult depressive symptoms. Carriers of the TT genotype who had been exposed to childhood physical abuse showed a mean BDI-II score of 17.4 compared with 10.0 for exposed subjects without the TT genotype. The effect of the primary analyses also survived Bonferroni correction for the testing of the five exposure dimensions. This result was confirmed for lifetime diagnosis of major depression according to DSM-IV. The lifetime prevalence of MDD was $23.9 \%$ in the exposed CC/CT carriers compared with $66.7 \%$ in the exposed TT carriers. In Figures 1 and 2 and Supplementary Table S1a, it is clearly indicated that the interaction effect between rs 1360780 and childhood physical abuse is represented by homozygote carriers of the T allele.

To explore the negative interaction effects in the other abuse and neglect dimensions, we increased the severity of childhood abuse and neglect to the most severe category. This 'severe' category is characterized by a higher frequency of traumatic experiences and/or more reported situations of abuse and neglect compared with the 'moderate' category. Although the number of affected subjects was further decreased, the results indicated that emotional and sexual abuse in the 'severe' category also interacted with rs1360780 ( $p<0.05$; Supplementary Table S3a). Comparing the group of subjects with at least one severe abuse category vs subjects with none, mild, moderate categories also demonstrated significant interaction with rs1360780 $(p=0.007$; Supplementary Table S4). These results indicate that all abuse dimensions with increasing severity contribute to the interaction effects. It is noteworthy that correlations between the five CTQ subdimensions were low to moderate $(r=0.11-0.56$; see Supplementary Table S5). The neglect dimension did not show a comparable interaction effect. Thus, the FKBP5 pathway seems to be more specific in mediating the psychobiological effects of abuse-related stress.

Our results clearly extend previous findings on the association of FKBP5 and mood disorders examined in clinical populations (Binder et al, 2004; Lekman et al, 2008). Especially when gene-environment interactions contribute to the pathophysiology of a distinct genetic marker, nonreplications of direct gene effects could occur because of low rates of childhood abuse exposure in the investigated samples (Gawlik et al, 2006; Papiol et al, 2007). Likewise, in our sample, no direct association between rs1360780 and depression was observed.

In secondary interaction analyses, we additionally investigated three intronic SNPs from the FKBP5 gene with different LDs $\left(r^{2}\right)$ to rs1360780: 0.09 for rs7757037, 0.41 for rs4713899, and 0.83 rs9368881 (Supplementary Figure S1). All four SNPs are widely distributed over the FKBP5 gene and cover different regions of the gene (Supplementary Figure S2). The less-linked SNP rs7757037 showed no evidence of interaction whereas the other two SNPs also indicated interactions with abuse dimension (Supplementary Table S1a). However, the AA genotype of rs4713899 included only one exposed subject. Thus, none of these two SNPs was superior to rs1360780.

Our finding could be of future relevance in the identification of subjects at high risk for depression after childhood abuse. Especially, the large effect sizes in BDI-II 
scores and in absolute risk of MDD suggest that the TT genotype of rs1360780 could be included in prediction models for subjects at high risk for depression. Based on the effect sizes in this study, a positive predictive value of $70 \%$ for the TT genotype is found in subjects with childhood physical abuse and prevalence of $30-35 \%$ of MDD (Supplementary Figure S4). However, the true value of a TT genotype screening in exposed subjects should be assessed longitudinally.

It is noteworthy that our results are in line with previous studies, associating the high-induction $\mathrm{T}$ allele of rs1360780 with an altered cortisol response to experimental stress (Luijk et al, 2010; Ising et al, 2008), with faster response to antidepressant treatment (Binder et al, 2004) and with the susceptibility to PTSD after childhood maltreatment (Xie et al, 2010; Binder et al, 2008). According to the pathophysiological model, the high-induction alleles of the FKBP5 gene are associated with a relative GR resistance (Binder et al, 2008; Binder, 2009), which could facilitate a long-lasting dysregulation of the HPA axis after childhood abuse and thereby increasing the risk for MDD.

As rs 1360780 was associated with the diagnoses of PTSD in former studies (Binder et al, 2008; Xie et al, 2010), analyses were also performed excluding all subjects with PTSD $(n=29)$ and adjusting for PTSD (see Supplementary Table S2). All interaction effects remained largely unchanged, indicating that our findings are valid for depression. To minimize a putative effect of cumulative adult traumatization on the interaction effects, we also adjusted all analyses for the number of traumatic events from the PTSD interview section. This adjustment did not change the interaction results. However, in both cases, PTSD and the number of adult traumata could be a causal mediator and no confounder (Rothman, 2002). In conclusion, PTSD and adult traumata did not account for the observed interaction effect between physical abuse, rs1360780, and depression in our study.

One has to discuss putative limitations of our study. The overall number of exposed TT carriers $(n=9)$ was small. To address this problem, statistically bootstrapping methods were applied for BDI-II scores. Moreover, to assess the effect of single observations on the statistic of interest, we performed detailed regression diagnostics for the diagnosis of MDD (Hosmer and Lemeshow, 2000). Four subjects with the largest impact on the statistical model were excluded separately each and all together from the model. Although the $95 \% \mathrm{CI}$ is wide, detailed model diagnostics indicate that the interaction itself was not dependent on a single observation (see Supplementary Tables S6 and S7). Moreover, the method applied is known for yielding relative large CIs, thus we have chosen a rather conservative approach (Nie et al, 2010).

In order to avoid major selection effects (eg, selected treatment samples) that may bias the results, we recruited the subjects from the general population (SHIP). From the baseline study (SHIP-0) that started in 1997, only $55.7 \%$ could be included in the LEGEND study (2007-2010). Importantly, we have no evidence that lifetime depressive symptoms at baseline had influenced the later participation in LEGEND.

One strength of our study is that we analyzed two outcomes of depression: a dimensional depression score
(BDI-II) and the lifetime diagnosis of major depression. There are obvious clinical differences between a self-report measure covering the last 2 weeks before the interview and an interview-based lifetime diagnosis of MDD (DSM-IV). We analyzed the statistical association between both end points. The area under the ROC curve was 0.69 , which indicates a weak relationship between BDI-II scores and MDD (Hosmer and Lemeshow, 2000). Thus, it is justified to consider MDD and BDI as two different measures.

In conclusion, our results support the role of the highinduction TT genotype of the rs1360780 polymorphism of the FKBP5 gene in the susceptibility to depressive disorders in the light of adverse, especially traumatic childhood events.

\section{ACKNOWLEDGEMENTS}

SHIP is part of the Community Medicine Research net of the University of Greifswald, Germany, which is funded by the Federal Ministry of Education and Research (Grants no. 01ZZ9603, 01ZZ0103, and 01ZZ0403), the Ministry of Cultural Affairs and the Social Ministry of the Federal State of Mecklenburg-West Pomerania. Genome-wide data have been supported by the Federal Ministry of Education and Research (Grant no. 03ZIK012) and a joint grant from Siemens Healthcare, Erlangen, Germany, and the Federal State of Mecklenburg-West Pomerania. The University of Greifswald is a member of the 'Center of Knowledge Interchange' program of the Siemens AG. This work was also funded by the German Research Foundation (DFG: GR 1912/5-1). HJG, KA, and CS had full access to all of the data in the study and take responsibility for the integrity of the data and the accuracy of the data analysis. We thank Daniela Becker, Varinia Popek, Elena Stoll, Frauke Grieme, Matthias Becker, Daniela Schrader, Julia Schwanda, Daniel Grams, and Andrea Rieck for their contribution to the study (organization, data collection, and data management).

\section{DISCLOSURE}

External financial support in the past 5 years: Hans Joergen Grabe: German Research Foundation; Federal Ministry of Education and Research Germany; speakers honoraria from Bristol-Myers Squibb, Eli Lilly, Novartis, Eisai, Wyeth, Pfizer, Boehringer Ingelheim, Servier; and travel funds from Janssen-Cilag, Eli Lilly, Novartis, AstraZeneca, and SALUSInstitute for Trend-Research and Therapy Evaluation in Mental Health. Carsten Spitzer: Travel funds and speakers honoraria from Janssen-Cilag and Boehringer Ingelheim; research grant from the 'Stiftung zur Aufarbeitung der SEDDiktatur.' Christian Schwahn, Katja Appel, Jessie Mahler, Andrea Schulz, Kristin Fenske, Jan Stender, Alexander Teumer: none. Sven Barnow: German Research Foundation; Federal Ministry of Health Germany. Ulrich John: German Research Foundation; German Cancer Aid; European Union; Federal Ministry of Education and Research Germany; Federal Ministry of Health; Social Ministry of the Federal State of Mecklenburg-West Pomerania of Germany. Matthias Nauck: research grants from the Federal Ministry of Education and Research Germany, BioRad Laboratories $\mathrm{GmbH}$, Siemens AG, Zeitschrift für 
Laboratoriumsmedizin, Bruker Daltronics, Abbott, Jurilab Kuopio, Roche Diagnostics, Dade Behring, DPC Biermann, and Becton Dickinson. Henry Völzke: research grants by Sanofi-Aventis, Biotronik, the Humboldt Foundation, the Federal Ministry of Education and Research (Germany), and the German Research Foundation. Reiner Biffar: research grants by Federal Ministry of Education and Research Germany, Ivoclar, Sirona, Dentsply, Kavo, Wieland Ceramics, GC, Heraeus, Dentaurum, Merz-Dental, the Krupp-Foundation, German Society of Dentistry (DGZMK), German Society of Prosthetic Dentistry and Dental Materials (DGZPW). Harald J Freyberger: German Research Foundation; Social Ministry of the Federal State of MecklenburgWest Pomerania; Family Ministry of the Federal Republic of Germany; speakers honoraria from AstraZeneca, Lilly, Novartis, and travel funds from Janssen-Cilag.

\section{REFERENCES}

American Psychiatric Association (1994). Diagnostic and Statistical Manual of Mental Disorders, 4th edn. American Psychiatric Press: Washington, DC.

Beck AT, Steer RA (1987). Beck Depression Inventory-Manual. The Psychological Corporation: San Antonio.

Bernstein DP, Stein JA, Newcomb MD, Walker E, Pogge D, Ahluvalia $\mathrm{T}$ et al (2003). Development and validation of a brief screening version of the Childhood Trauma Questionnaire. Child Abuse Negl 27: 169-190.

Binder EB (2009). The role of FKBP5, a co-chaperone of the glucocorticoid receptor in the pathogenesis and therapy of affective and anxiety disorders. Psychoneuroendocrinology 34(Suppl 1): S186-S195.

Binder EB, Bradley RG, Liu W, Epstein MP, Deveau TC, Mercer KB et al (2008). Association of FKBP5 polymorphisms and childhood abuse with risk of posttraumatic stress disorder symptoms in adults. JAMA 299: 1291-1305.

Binder EB, Salyakina D, Lichtner P, Wochnik GM, Ising M, Putz B et al (2004). Polymorphisms in FKBP5 are associated with increased recurrence of depressive episodes and rapid response to antidepressant treatment. Nat Genet 36: 1319-1325.

Bradley RG, Binder EB, Epstein MP, Tang Y, Nair HP, Liu W et al (2008). Influence of child abuse on adult depression: moderation by the corticotropin-releasing hormone receptor gene. Arch Gen Psychiatry 65: 190-200.

Carpenter LL, Tyrka AR, McDougle CJ, Malison RT, Owens MJ, Nemeroff CB et al (2004). Cerebrospinal fluid corticotropinreleasing factor and perceived early-life stress in depressed patients and healthy control subjects. Neuropsychopharmacology 29: 777-784.

Caspi A, Sugden K, Moffitt TE, Taylor A, Craig IW, Harrington H et al (2003). Influence of life stress on depression: moderation by a polymorphism in the 5-HTT gene. Science 301: 386-389.

Gawlik M, Moller-Ehrlich K, Mende M, Jovnerovski M, Jung S, Jabs $B$ et al (2006). Is FKBP5 a genetic marker of affective psychosis? A case control study and analysis of disease related traits. BMC Psychiatry 6: 52.

Grabe HJ, Lange M, Wolff B, Völzke H, Lucht M, Freyberger HJ et al (2005). Mental and physical distress is modulated by a polymorphism in the 5-HT transporter gene interacting with social stressors and chronic disease burden. Mol Psychiatry 10: 220-224.

Grabe HJ, Schwahn C, Appel K, Mahler J, Schulz A, Spitzer C et al (2011). Update on the 2005 paper: moderation of mental and physical distress by polymorphisms in the 5-HT transporter gene by interacting with social stressors and chronic disease burden. Mol Psychiatry 16: 354-356.
Grabe HJ, Spitzer C, Schwahn C, Marcinek A, Frahnow A, Barnow S et al (2009). Serotonin transporter gene (SLC6A4) promoter polymorphisms and the susceptibility to posttraumatic stress disorder in the general population. Am J Psychiatry 166: $926-933$.

Greenland S (2008). Introduction to regression models. In: Rothman $\mathrm{KJ}$ et al (eds). Modern Epidemiology. Lippincott Williams \& Wilkins: Philadelphia. pp 381-417.

Greenland S, Lash TL, Rothman KJ (2008). Concepts of interaction. In: Rothman KJ, et al (eds). Modern Epidemiology. Lippincott Williams \& Wilkins: Philadelphia. pp 71-83.

Harrell Jr FE (2001). Regression Modeling Strategies. With Applications to Linear Models, Logistic Regression, and Survival Analysis. Springer: New York.

Heim C, Nemeroff CB (2001). The role of childhood trauma in the neurobiology of mood and anxiety disorders: preclinical and clinical studies. Biol Psychiatry 49: 1023-1039.

Holsboer F (1999). The rationale for corticotropin-releasing hormone receptor (CRH-R) antagonists to treat depression and anxiety. $J$ Psychiatr Res 33: 181-214.

Holsboer F (2000). The corticosteroid receptor hypothesis of depression. Neuropsychopharmacology 23: 477-501.

Hosmer DW, Lemeshow S (2000). Applied Logistic Regression. Wiley: New York.

Ising M, Depping AM, Siebertz A, Lucae S, Unschuld PG, Kloiber S et al (2008). Polymorphisms in the FKBP5 gene region modulate recovery from psychosocial stress in healthy controls. Eur $J$ Neurosci 28: 389-398.

John U, Greiner B, Hensel E, Ludemann J, Piek M, Sauer S et al (2001). Study of Health In Pomerania (SHIP): a health examination survey in an East German region: objectives and design. Soz Praventivmed 46: 186-194.

Lekman M, Laje G, Charney D, Rush AJ, Wilson AF, Sorant AJ et al (2008). The FKBP5-gene in depression and treatment responsean association study in the Sequenced Treatment Alternatives to Relieve Depression (STAR $\left.{ }^{\star} \mathrm{D}\right)$ Cohort. Biol Psychiatry 63: 1103-1110.

Luijk MP, Velders FP, Tharner A, van Ijzendoorn MH, Bakermans-Kranenburg MJ, Jaddoe VW et al (2010). FKBP5 and resistant attachment predict cortisol reactivity in infants: gene-environment interaction. Psychoneuroendocrinology 35: 1454-1461.

Nie L, Chu H, Li F, Cole SR (2010). Relative excess risk due to interaction: resampling-based confidence intervals. Epidemiology 21: 552-556.

Papiol S, Arias B, Gasto C, Gutierrez B, Catalan R, Fananas L (2007). Genetic variability at HPA axis in major depression and clinical response to antidepressant treatment. J Affect Disord 104: 83-90.

Persons JB, Perloff JM (1989). The relationship between attributions and depression varies across attributional dimensions and across samples. J Psychopathol Behav Assess 11: 47-60.

Rothman KJ (2002). Epidemiology: An Introduction. Oxford University Press: New York.

Roy A, Gorodetsky E, Yuan Q, Goldman D, Enoch MA (2010). Interaction of FKBP5, a stress-related gene, with childhood trauma increases the risk for attempting suicide. Neuropsychopharmacology 35: 1674-1683.

Team RDC (2010). R: A Language and Environment for Statistical Computing. R Foundation for Statistical Computing: Vienna, Austria.

Vermeer H, Hendriks-Stegeman BI, van der Burg B, van Buul-Offers SC, Jansen M (2003). Glucocorticoid-induced increase in lymphocytic FKBP51 messenger ribonucleic acid expression: a potential marker for glucocorticoid sensitivity, potency, and bioavailability. J Clin Endocrinol Metab 88: 277-284. 
Völzke H, Alte D, Schmidt CO, Radke D, Lorbeer R, Friedrich N et al (2011). Cohort profile: the Study of Health in Pomerania. Int J Epidemiol 40: 294-307.

Wingenfeld K, Spitzer C, Mensebach C, Grabe H, Hill A, Gast $U$ et al (2010). The German version of the Childhood Trauma Questionnaire (CTQ): preliminary psychometric properties. Psychother Psychosom Med Psychol 60: 442-450.

Wittchen H, Höfler M, Gander F, Pfister H, Storz S, Üstün B et al (1999). Screening for mental disorders: performance of the Composite International Diagnostic-Screener (CID-S). Int J Meth Psychiat Res 8: 59-70.

Wittchen H-U, Pfister H (1997). Diagnostisches Expertensystem für psychische Störungen. Swets \& Zeitlinger: Frankfurt.
Wochnik GM, Ruegg J, Abel GA, Schmidt U, Holsboer F, Rein T (2005). FK506-binding proteins 51 and 52 differentially regulate dynein interaction and nuclear translocation of the glucocorticoid receptor in mammalian cells. J Biol Chem 280: 4609-4616.

Xie P, Kranzler HR, Poling J, Stein MB, Anton RF, Farrer LA et al (2010). Interaction of FKBP5 with childhood adversity on risk for post-traumatic stress disorder. Neuropsychopharmacology 35: $1684-1692$.

Zobel AW, Nickel T, Sonntag A, Uhr M, Holsboer F, Ising M (2001). Cortisol response in the combined dexamethasone/CRH test as predictor of relapse in patients with remitted depression. A prospective study. J Psychiatr Res 35: 83-94.

Zou GY (2008). On the estimation of additive interaction by use of the four-by-two table and beyond. Am J Epidemiol 168: 212-224.

Supplementary Information accompanies the paper on the Neuropsychopharmacology website (http://www.nature.com/npp) 